\title{
Investigación original \\ Diseño, Implementación y Evaluación de un Servicio de Educación Sanitaria Sobre el Uso Correcto de los Medicamentos en un Hospital Pediátrico Mexicano
}

\author{
Design, implementation and assessment of a health education service on the correct use \\ of drugs in a pediatric Mexican hospital \\ Sandra RIVERA, Maricela LOPEZ OROZCO.
}

\begin{abstract}
RESUMEN *
Se implementó un Servicio de Educación Sanitaria, en un Hospital Pediátrico Mexicano (Hospital del Niño DIF-Hidalgo), como servicio dependiente de los Servicios Farmacéuticos Universitarios ubicados en el hospital. El principal objetivo de este servicio es el de proporcionar información objetiva, independiente, actualizada y evaluada de manera sencilla, clara, concisa y precisa, sobre el Uso Correcto de los Medicamentos a los padres o tutores de los pacientes egresados, familiares y a la comunidad en general. La evaluación del Servicio, se realizó al comparar el grado de conocimiento de los padres o tutores de los pacientes egresados, antes y después de una sesión educativa por parte del farmacéutico, se hizo la evaluación, mediante dos entrevistas, a 270 padres o tutores, la entrevista midió el grado de conocimiento de: 1)la patología del paciente, 2) la definición de medicamento y efecto secundario, 3) tratamiento farmacológico y no farmacológico. El estudio demostró que los padres o tutores aumentaron el grado de conocimiento sobre la enfermedad y terapéutica de los pacientes, después de recibir educación sanitaria $(p<0.001)$.
\end{abstract}

Palabras clave: Educación sanitaria. Servicios farmacéuticos. Grado de conocimientos

\begin{abstract}
A Service of Health Education was implemented, in a Mexican Pediatric Hospital (Hospital del Niño DIF-Hidalgo), as dependent service of the Hospital Pharmacy Services, being the main objective of the service to provide independent, up-to-date and evaluated information in a simple, clear, concise and precise format, on the Correct Use of the Medications to the parents or tutors of the patient, family and to the community in general. Comparing the patient parents 'or tutors' awareness, before and after an educational session pharmacist provided carried out the evaluation of the Service. The evaluation was made, through two interviews to 270 parents or tutors. The interview measured the patient knowledge about: 1) illness, 2) the medication definition and adverse effects, 3 ) pharmacological and not pharmacological treatment. The study demonstrated that the parents or tutors significantly increased the knowledge on the illness and the patients' therapy, after receiving health education $(\mathrm{p}<0.001)$.
\end{abstract}

Keywords: Health education. Pharmacy services. Awareness.

\section{(Español)}

\section{INTRODUCCIÓN}

La Educación Sanitaria sobre el Uso de los Medicamentos, plantea como objetivos el crear una actitud crítica hacia los medicamentos como solución universal a los problemas de salud, y hacia la publicidad de medicamentos "milagrosos", los pacientes deben recibir información actualizada e individualizada sobre su medicación, definir grupos de acción prioritaria para educación sanitaria sobre medicamentos e identificar sus necesidades específicas. La educación sanitaria sobre medicamentos debe integrarse de forma planificada en la totalidad del sistema de salud y en los programas nacionales de educación sanitaria. ${ }^{1-3}$ Siendo estos los principales objetivos, sin embargo ante una patología y un tratamiento específico, el objetivo activo debe ser la obtención de una cooperación activa del paciente con el fin de aumentar el grado de cumplimiento y adhesión a la
*Sandra RIVERA. Licenciada en Farmacia. Área Académica de Farmacia. Instituto de Ciencias de la Salud. Universidad Autónoma del Estado de Hidalgo. Farmacéutico del Hospital del Niño DIF. México.

Maricela LOPEZ OROZCO. Maestro en Ciencias. Área Académica de Farmacia. Instituto de Ciencias de la Salud. Universidad Autónoma del Estado de Hidalgo. México. 
prescripción, disminuir el número de errores de dosificación, horarios y técnicas de administración. ${ }^{4-}$

El objetivo, de este trabajo fue el diseñar, implementar y evaluar un Servicio de Educación Sanitaria dirigido a los pacientes egresados en un Hospital Pediátrico Mexicano, sobre el Uso Correcto de los Medicamentos.

\section{MÉTODOS}

Se diseño del Servicio de Educación, se realizó para un Hospital Pediátrico Mexicano, el cual es de segundo nivel de atención y cuenta con los servicios de:

A) Consulta externa en pediatría y B) Hospitalización: Unidad de Cuidados Intensivos Neonatales (UCIN), Lactantes, Medicina Interna, Infectología, Cirugía, Unidad de Cuidados Intensivos (UTIP). El hospital del Niño DIF tiene 62 camas. Brinda atención médica a pacientes pediátricos de 0 a 16 años de edad.

Para el diseño del Servicio se utilizó la siguiente metodología: 1) identificación del grupo de población con necesidades informativas prioritarias sobre el uso de medicamento, 2) evaluación de las necesidades de información de la población seleccionada, 3) planificación de las funciones del servicio, 4) implementación del servicio, 5) evaluación del servicio.

En la Evaluación del servicio, se realizó un estudio de tipo intervención - prospectivo en un periodo de 9 meses, estó mediante la comparación del aumento del conocimiento de los padres y/o tutores de los pacientes egresados, antes y después de una sesión educativa por parte de los farmacéuticos del hospital. Se realizaron 270 entrevistas, las cuales midieron el aumento del conocimiento de: 1) patología del paciente, 2) definición de medicamento y efecto secundario, 3) tratamiento farmacológico y no farmacológico.

Las variables que se estudiaron y se presentan en cuadro en forma individual:

1) Nivel de escolaridad de los padres y/o tutores de los pacientes

- Primaria inconclusa o terminada

- Secundaria inconclusa o terminada

- Nivel medio superior

- Nivel superior

2) Conocimiento de la patología

- ¿Conoce la enfermedad que padece su hijo(a)?

- ¿Sabe cuáles son las manifestaciones de la enfermedad de su hijo(a)?

- Sabe por cuánto tiempo estará enfermo su hijo (a)?

3) Conocimiento sobre definiciones

- ¿Sabe qué es un medicamento?

- ¿Sabe qué es una efecto secundario?

4) Conocimiento del tratamiento farmacológico y no farmacológico
- ¿Sabe por cuánto tiempo va a tomar el o los medicamentos su hijo(a)?

- ¿Sabe cuántos medicamentos tiene que tomar su hijo (a)?

- ¿Sabe cuáles los riesgos de tomar otros medicamentos que no le haya sugerido por su médico?

- ¿ldentifica los medicamentos del tratamiento?

- ¿Conoce el tratamiento no farmacológico?

El análisis de los resultados de la evaluación, se realizó mediante la prueba de McNemar, la cual es una prueba no paramétrica para relacionar dos variables de tipo dicotómicas. Útil para detectar cambios de respuestas en diseños experimentales de tipo intervención en "antes y después", utiliza una distribución de chi-cuadrada para cambios de respuesta. Un valor de $p<0.01$ se consideró significativo. Para la aplicación de la prueba de McNemar en los resultados obtenidos se utilizó el programa SPSS 8.0 versión Windows.

\section{RESULTADOS Y DISCUSIÓN}

El diseño del Servicio de Educación se propuso a la Dirección General del Hospital del Niño DIFHidalgo, siendo aceptada su implementación e integrándose como un servicio dependiente de los Servicios Farmacéuticos del Hospital.

La implementación del servicio se realizó en dos fases: Primer fase: educación al paciente egresado y elaboración de material educativo. Segunda fase: sistema de seguimiento del cumplimiento en pacientes crónicos.

Considerando los resultados obtenidos sobre la implementación del Servicio en el primer año de funcionamiento, se decidió desarrollar la evaluación del mismo. Dicha evaluación estudió el grado de conocimiento de los padres o tutores de los pacientes egresados del Hospital, sobre la enfermedad y terapéutica de sus hijos antes y después de recibir una sesión educativa por parte del Servicio de Educación.

\begin{tabular}{|l|c|c|}
\hline \multicolumn{3}{|c|}{$\begin{array}{l}\text { Tabla 1. ¿Conoce la enfermedad que padece su } \\
\text { hijo(a)? }\end{array}$} \\
\hline & Antes & Después \\
\hline Sí & 136 & 229 \\
\hline No & 134 & 41 \\
\hline $\mathrm{n}=270$ & $\mathrm{X} 2=91.011$ & $\mathrm{P}<0.001$ \\
\hline
\end{tabular}

Uno de los principales factores que se observó fue que la mayor parte de la población entrevistada tenía un bajo nivel de escolaridad (figura 1), por lo cual, se consideró que existe una asociación entre el nivel de escolaridad y el grado de conocimiento de la enfermedad y terapéutica de los padres o tutores de los pacientes.

En relación al conocimiento de la enfermedad se considera importante que los padres o tutores de los pacientes conozcan cual es la enfermedad de su hijo(a), con el fin de que entiendan la importancia de cumplir el tratamiento farmacológico y no farmacológico. En la tabla I se observa que los padres o tutores incrementaron su conocimiento 
sobre la enfermedad que tenían sus hijos al momento del egreso, con relación a antes y después de la sesión educativa, esta diferencia fue estadísticamente significativa $(p<0.001)$.

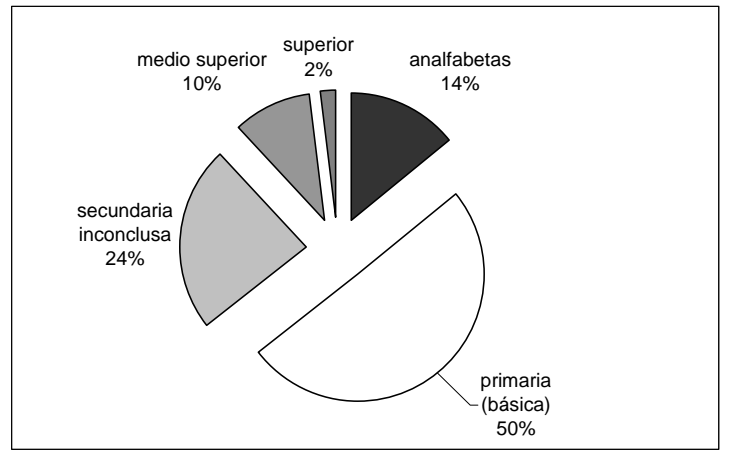

Figura 1. Nivel de Escolaridad de padres y/o tutotes.

Así mismo, se observo que la mitad de la población entrevistada no conocían las manifestaciones de la enfermedad y 198 de los padres o tutores desconocían la duración de la misma antes de la intervención del farmacéutico (tabla 2 y 3), lo cual posiblemente se debió a que no existió una adecuada relación profesional del área de la salud - paciente, así como al nivel de escolaridad de los mismos pudo ser un factor que se vio reflejado en el desconocimiento de la enfermedad del paciente. Sin embargo, después de la intervención del farmacéutico, disminuyó el número de padres o tutores que desconocían ambos aspectos, lo cual demuestra que las sesiones educativas incrementaron el grado de conocimiento de la enfermedad.

\begin{tabular}{|l|l|l|}
\hline \multicolumn{3}{|l|}{$\begin{array}{l}\text { Tabla 2. ¿Sabe cuáles son las manifestaciones } \\
\text { de la enfermedad de su hijo(a)? }\end{array}$} \\
\hline & Antes & Después \\
\hline Sí & 133 & 225 \\
\hline No & 137 & 45 \\
\hline $\mathrm{n}=270$ & $\mathrm{X} 2=90.001$ & $\mathrm{P}<0.001$ \\
\hline
\end{tabular}

\begin{tabular}{|c|c|c|}
\hline \multicolumn{3}{|c|}{$\begin{array}{l}\text { Tabla 3. ¿Sabe por cuánto tiempo estará enfermo } \\
\text { su hijo (a)? }\end{array}$} \\
\hline & Antes & Después \\
\hline Sí & 72 & 227 \\
\hline No & 198 & 43 \\
\hline$n=270$ & $X 2=153.006$ & $P<0.001$ \\
\hline
\end{tabular}

Cabe destacar que un reducido número de padres o tutores manejaban los conceptos o definiciones de efecto secundario y medicamento (tabla 4 y 5 ), además los efectos secundarios frecuentemente los adjudicaron a molestias o efectos propios de la enfermedad y una gran parte consideraban a los medicamentos como productos milagrosos, sin saber que los medicamentos controlan en muchas ocasiones la enfermedad de su hijo(a). A pesar de que se demostró estadísticamente, que aumentó el conocimiento de estos conceptos, 79 de los entrevistados, no comprendieron en su totalidad el significado de medicamento y 128 del total el concepto de efecto secundario, después de la sesión educativa.

\begin{tabular}{|l|l|l|}
\hline \multicolumn{3}{|c|}{ Tabla 4. ¿Sabe qué es un medicamento? } \\
\hline & Antes & Después \\
\hline Sí & 27 & 191 \\
\hline No & 243 & 79 \\
\hline $\mathrm{n}=270$ & $\mathrm{X} 2=162.006$ & $\mathrm{P}<0.001$ \\
\hline
\end{tabular}

\begin{tabular}{|l|l|l|}
\hline \multicolumn{3}{|c|}{ Tabla 5. ¿Sabe qué es una efecto secundario? } \\
\hline & Antes & Después \\
\hline Sí & 13 & 142 \\
\hline No & 257 & 128 \\
\hline $\mathrm{n}=270$ & $\mathrm{X} 2=127.008$ & $\mathrm{P}<0.001$ \\
\hline
\end{tabular}

En relación al conocimiento del tratamiento farmacológico y no farmacológico que debe seguir su hijo(a) después de su egreso hospitalario, y que son aspectos importantes para fomentar el cumplimiento del tratamiento, se observó que antes de la sesión educativa 88 entrevistados, conocían la duración del tratamiento y 117 conocía el número de medicamentos prescritos y los identificaba. Después de la intervención de los farmacéuticos como educadores, se demostró un aumento significativo en el grado del conocimiento de ambos aspectos.

Durante las sesiones educativas, se alertó a los padres o tutores de los pacientes sobre los riesgos de la misma y se observó que antes de la sesión educativa, 66 (24.44\%)de los entrevistados desconocían los riesgos de consumir medicamentos no prescritos por el médico y después de recibir la sesión educativa 218 $(80.74 \%)$ de los padres o tutores ya conocían los riesgos de la automedicación.

Con respecto al grado de conocimiento, sobre el tratamiento no farmacológico que se debería de seguir posterior al egreso, sólo 43 padres o tutores conocían este aspecto antes de la sesión educativa y después de haberla recibido el número, de padres o tutores que conocían el tratamiento no farmacológico, se incrementó.

Si bien, existió un aumento importante en el conocimiento de los padres o tutores sobre el tratamiento farmacológico y no farmacológico, lo cual se demostró con una significación estadística $(p<0.001$ ), para cada aspecto medido (tabla 6,7 , $8,9,10)$, se considera importante que éstos incrementen su conocimientos sobre la identificación de los medicamentos, así como, la duración del tratamiento, al $100 \%$, ya que son aspectos sumamente importantes para que el paciente cumpla con el tratamiento.

\begin{tabular}{|c|c|c|}
\hline \multicolumn{3}{|c|}{$\begin{array}{l}\text { Tabla 6. ¿Sabe por cuánto tiempo va a tomar el o } \\
\text { los medicamentos su hijo(a)? }\end{array}$} \\
\hline & Antes & Después \\
\hline Sí & 88 & 257 \\
\hline No & 182 & 13 \\
\hline $\mathrm{n}=270$ & $\mathrm{X}^{2}=167.006$ & $\mathrm{p}<0.001$ \\
\hline
\end{tabular}




\begin{tabular}{|c|c|c|}
\hline \multicolumn{3}{|c|}{$\begin{array}{l}\text { Tabla 7. ¿Sabe cuántos medicamentos tiene que } \\
\text { tomar su hijo (a)? }\end{array}$} \\
\hline & Antes & Después \\
\hline Sí & 117 & 268 \\
\hline No & 153 & 2 \\
\hline $\mathrm{n}=270$ & $\mathrm{X}^{2}=149.007$ & $\mathrm{p}<0.001$ \\
\hline
\end{tabular}

\begin{tabular}{|c|c|c|}
\hline \multicolumn{3}{|c|}{$\begin{array}{l}\text { Tabla 8. ¿Sabe cuáles los riesgos de tomar otros } \\
\text { medicamentos que no le haya sugerido por su } \\
\text { médico? }\end{array}$} \\
\hline & Antes & Después \\
\hline Sí & 66 & 218 \\
\hline No & 204 & 52 \\
\hline $\mathrm{N}=270$ & $X^{2}=150.007$ & $\mathrm{p}<0.001$ \\
\hline
\end{tabular}

\begin{tabular}{|c|c|c|}
\hline \multicolumn{3}{|c|}{$\begin{array}{l}\text { Tabla 9. ¿Identifica los } \\
\text { tratamiento? }\end{array}$} \\
\hline & Antes & Después \\
\hline Sí & 117 & 264 \\
\hline No & 153 & 6 \\
\hline$n=270$ & $X^{2}=145.007$ & $\mathrm{p}<0.001$ \\
\hline
\end{tabular}

\begin{tabular}{|c|c|c|}
\hline \multicolumn{3}{|c|}{$\begin{array}{l}\text { Tabla 10. ¿Conoce el tratamiento } \\
\text { farmacológico? }\end{array}$} \\
\hline & Antes & Después \\
\hline Sí & 43 & 203 \\
\hline No & 227 & 67 \\
\hline $\mathrm{n}=270$ & $X^{2}=158.006$ & $\mathrm{p}<0.001$ \\
\hline
\end{tabular}

Esto último, nos lleva a modificar las estrategias en la educación al paciente egresado del Servicio de Educación Sanitaria, reforzando las sesiones educativas sobre el tratamiento farmacológico y no farmacológico con instrucciones por escrito, folletos, tarjetas recordatorias, dibujos (en caso de padres analfabetas), etc. Estas estrategias han sido efectivas en diversos trabajos publicados.

Otro aspecto importante que se debe señalar es la automedicación y riesgos de tomar medicamentos no prescritos por el médico, lo cual debe de ser considerado como un problema que se debe abordar no sólo por el farmacéutico, sino por todo el equipo salud en general, implementando mecanismos más efectivos que regulen el consumo de medicamentos. En el estudio, se demostró que aumentó considerablemente el conocimiento sobres los riesgos de la automedicación, después de la sesión educativa impartida por los farmacéuticos, lo cual nos demuestra que el farmacéutico puede convertirse en un elemento eficaz en la Atención Primaria de Salud, al contrarrestar, entre otras cosas, los riesgos de la automedicación.

\section{CONCLUSION}

De acuerdo a los resultados se puede concluir que:

1. El grado de conocimiento inicial de los padres o tutores sobre la enfermedad y terapéutica de sus hijos es muy deficiente.

2. Mediante la intervención del Servicio de Educación Sanitaria se incrementó el grado de conocimiento de los padres o tutores sobre la enfermedad y terapéutica que debe seguir su hijo(a) después de su egreso hospitalario, y por lo tanto, se fomentó el Uso Correcto de los Medicamentos.

3. El Servicio logró corregir algunas interpretaciones erróneas que lo padres o tutores tenían sobre la terapéutica y la enfermedad de sus niños, lo cual ayudará a fomentar el cumplimiento del tratamiento en los pacientes después del egreso hospitalario.

4. Este Servicio puede ayudar a mejorar el Uso de los Medicamentos en el estado de Hidalgo y fomentar una cultura en torno al uso correcto de los mismos.

\section{Referencias}

1 Bonal, J. y Domínguez, A. (1992): Farmacia Hospitalaria. $2^{\circ}$ edición. EMISA. 560-564.

2. Faus, Ma. J y Martínez, F. (1999): La atención farmacéutica en Farmacia comunitaria: evolución de conceptos, necesidades de formación, modalidades y estrategias para su puesta en marcha. Pharmacéutical Care. 1:52-61.

3. Max, D. (1998): Shared borders: Achieving the goals of interdiciplinary patient care. Am J Health - Syst Pharm.55:13691374.

4. Burgess, M. (1989): Ethical and economic aspects of noncompliance and overtreatment. Can Med Assoc J. 141:777-780.

5. McKenney, J. y Harrison, W. (1976): Drug related hospital admissions. Am J Hosp Pharm. 33:792-795.

6. Ruiz, J. (1990): Motivación del paciente y cumplimiento del régimen terapéutico. Medicina Integral. 40:114-116.

7. Hepler, C.D., y Strand, L.M. (1999): Oportunidades y responsabilidades en la Atención Farmacéutica. Pharm. Care. 1:3547.

8. ASHP. (1993): Guidelines on pharmacy conducted patient counseling. Am J Hosp. Pharm. 50:505-506.

9. Prieto, V. Zúñiga, O.C. Vargas, C .y Domecq, J. (1994): Análisis del impacto de un programa educativo en pacientes cardiópatas. OFIL. 4(1):24-28

10. Genua, M.I. Sanzberro, E. y Gutiérrez, L. (1991): Análisis del grado de cumplimiento y de conocimiento del tratamiento de pacientes ingresados en un Servicio de Medicina. OFIL. 1(1): 2-10

11. Canales, F., Alvarado, E.L., y Pineda, E. B. (2000): Metodología de investigación. Manual para el desarrollo de personal de Salud. UTEHA. México. 